\title{
Tachyon field non-minimally coupled to massive neutrino matter
}

\author{
Safia Ahmad ${ }^{a}$ Nurgissa Myrzakulov $^{b}$ R. Myrzakulov ${ }^{b}$ \\ ${ }^{a}$ Centre for Theoretical Physics, Jamia Millia Islamia, \\ New Delhi-110025, India \\ ${ }^{b}$ Eurasian International Center for Theoretical Physics, Eurasian National University, \\ Astana 010008, Kazakhstan \\ E-mail: safia@ctp-jamia.res.in,nmyrzakulov@gmail.com,rmyrzakulov@gmail.com
}

\begin{abstract}
In this paper, we consider rolling tachyon, with steep run-away type of potentials non-minimally coupled to massive neutrino matter. The coupling dynamically builds up at late times as neutrino matter turns non-relativistic. In case of scaling and string inspired potentials, we have shown that non-minimal coupling leads to minimum in the field potential. Given a suitable choice of model parameters, it is shown to give rise to late-time acceleration with the desired equation of state.
\end{abstract}

Keywords: dark energy theory, string theory and cosmology 


\section{Contents}

1 Introduction $\quad 1$

2 Rolling tachyon and late time dynamics $\quad 2$

$\begin{array}{lll}2.1 & \text { Observational Constraints } & 7\end{array}$

3 Cosmology with string inspired Potential 9

4 Conclusion

\section{Introduction}

Since the discovery of late-time cosmic acceleration in 1998 [1-3], model building was undertaken in cosmology to capture this remarkable phenomenon. To this effect, a variety of scalar field models including quintessence [4-16], phantom field [17-21], rolling tachyon [22-25] and others were investigated in the literature. This description, however, has limited predictive power. Indeed, for a priori given cosmic history, one can always construct a field potential which would give rise to that history. However, cosmological dynamics of scalar field may still be of interest if the field has generic features such as tracking behavior $[4,5]$ or the field is inspired by a fundamental theory of high energy physics. For instance, rolling tachyon, inspired by string theory, was taken up with great interest in cosmology few years back. The string inspired potential for rolling tachyon is typically run-away type. It behaves like decaying exponential away from the maximum such that dark matter is a late-time attractor of the dynamics [26-44]. Following work of Sen [22, 23], efforts were made to obtain inflation [45-84] using rolling tachyon. Though there is region of slow-roll near the top of the potential, one could not collect enough number of e-folds; there is no adjustable parameter in the potential (see also Ref. [85] on the related theme).

Following the initial development, cosmologists investigated phenomenologically a number of potentials in the framework of rolling tachyon. For instance, it was shown that $V(\phi) \sim \phi^{-2}$, an analog of exponential potential for standard quintessence, gives rise to scaling solution as an attractor of the dynamics $[4,5]$. The equation of state parameter for rolling tachyon is given by, $\omega_{\phi}=\left(\dot{\phi}^{2}-1\right)$ which remains constant during scaling regime such that $\omega_{\phi}=\omega_{b}[26,27,86,87]$. Thus, it requires background fluid with negative equation of state $\omega_{b}$ thereby the scaling solution can not track the standard background(matter/radiation) in this case. Be it the string inspired case or the scaling potential, they can not account for late-time acceleration. It was demonstrated in Ref. [87] that coupling of tachyon with matter gives rise to scaling solution which is accelerating and mimics standard matter.

During slow-roll, rolling tachyon dynamics reduces to canonical description. Coupling to matter then induces minimum in the potential. For large value of the coupling, minimum of the potential is more pronounced and the field can easily be trapped there mimicking cosmological constant like behavior. Unfortunately, in this case, the attractor is reached once matter phase is established which is not a desirable feature; the matter phase should be left intact.

The above mechanism based upon non-minimal coupling may be salvaged by invoking non-minimal coupling with massive neutrino matter [88-111] leaving the standard matter 
minimally coupled. We should admit that this is purely a phenomenological setting which looks attractive for the following reason. Since neutrinos become non-relativistic at late times, the coupling builds up dynamically at late stages. The late-time acceleration gets associated with the said physical phenomenon.

In this paper, we shall study rolling tachyon, with scaling and string inspired potentials, assuming non-minimal coupling to massive neutrino matter and examine the possibility of late time acceleration in these cases.

\section{Rolling tachyon and late time dynamics}

We shall consider a situation where a tachyon field $\phi$, with an equation of state $\omega_{\phi} \equiv p_{\phi} / \rho_{\phi}$, is coupled non-minimally to massive neutrino matter with an equation of state $\omega_{\nu} \equiv p_{\nu} / \rho_{\nu}$. The continuity equations for $\rho_{\phi}$ and $\rho_{\nu}$ in the spatially flat Friedmann-Robertson-Walker (FRW) background are [112]

$$
\begin{gathered}
\dot{\rho_{\phi}}+3 H\left(\rho_{\phi}+p_{\phi}\right)=-Q \sqrt{V}\left(\rho_{\nu}-3 p_{\nu}\right) \frac{\dot{\phi}}{M_{\mathrm{Pl}}}, \\
\dot{\rho_{\nu}}+3 H\left(\rho_{\nu}+p_{\nu}\right)=Q \sqrt{V}\left(\rho_{\nu}-3 p_{\nu}\right) \frac{\dot{\phi}}{M_{\mathrm{Pl}}},
\end{gathered}
$$

where $\mathrm{Q}$ is the coupling between the tachyon field and the neutrino matter [113], $M_{\mathrm{Pl}}$ is the reduced Planck mass and dots are the derivatives with respect to the time $t$.

The Lagrangian of a tachyon field is given by

$$
p_{\phi}=-V(\phi) \sqrt{1-\dot{\phi}^{2}}
$$

where we have chosen an inverse square potential, $V(\phi)=4 M_{\mathrm{Pl}}^{2} /\left(\lambda^{2} \phi^{2}\right)$. Here $\lambda$, which is related to the slope of the potential, can be determined for scaling solution, i.e. when $\rho_{\phi} / \rho_{\nu}=$ const, by following the same procedure as in $[86,112]$ and taking into account the change in the total fractional density at late times for the system being considered here, which is $\Omega_{m}+\Omega_{\phi}+\Omega_{\nu}=1$,

$$
\lambda \equiv-M_{\mathrm{Pl}} \frac{V^{\prime}}{V^{3 / 2}}=Q \frac{\left(1-3 \omega_{\nu}\right)\left(\Omega_{\nu}\left(1+\omega_{\nu}\right)+\Omega_{\phi}\left(1+\omega_{\phi}\right)\right)}{\Omega_{\phi}\left(\omega_{\nu}-\omega_{\phi}\right)}
$$

where, $\Omega_{\phi}$ is the tachyon field density parameter.

The energy density for the field $\phi$ corresponding to the Lagrangian (2.3) is

$$
\rho_{\phi}=\frac{V(\phi)}{\sqrt{1-\dot{\phi}^{2}}}
$$

Equation of state parameter for tachyon field is therefore,

$$
\omega_{\phi} \equiv \frac{p_{\phi}}{\rho_{\phi}}=\dot{\phi}^{2}-1
$$

In the spatially flat Friedmann-Robertson-Walker background, we have the following Friedmann equations,

$$
\begin{aligned}
3 H^{2} M_{\mathrm{Pl}}^{2} & =\rho_{m}+\rho_{r}+\rho_{\phi}+\rho_{\nu}, \\
\left(2 \dot{H}+3 H^{2}\right) M_{\mathrm{Pl}}^{2} & =V(\phi) \sqrt{1-\dot{\phi}^{2}}-\omega_{\nu} \rho_{\nu}
\end{aligned}
$$


Here we have used the standard continuity equations of radiation and non-relativistic matter (excluding massive neutrino matter which couples directly to the tachyon field),

$$
\begin{array}{r}
\dot{\rho_{m}+3 H \rho_{m}}=0 \\
\dot{\rho}_{r}+4 H \rho_{r}=0
\end{array}
$$

The equation of motion for the tachyon field $\phi$ can then be obtained by using the eqns. $(2.1),(2.5)$ and (2.6),

$$
\frac{\ddot{\phi}}{1-\dot{\phi}^{2}}+3 H \dot{\phi}+\frac{V^{\prime}}{V}=-\frac{\sqrt{1-\dot{\phi}^{2}}}{\sqrt{V} M_{\mathrm{Pl}}} Q\left(\rho_{\nu}-3 p_{\nu}\right)
$$

with the equation-of-state parameter for the neutrino matter defined in such a way that neutrinos are relativistic for most of the history of the universe and become non-relativistic in the recent times [110],

$$
\omega_{\nu}=\frac{1}{6}\left[1+\tanh \left(\frac{\ln (1+z)-z_{\mathrm{eq}}}{z_{\mathrm{dur}}}\right)\right]
$$

where the epoch around which the transition of neutrino from relativistic to non-relativistic occurs, is determined by $z_{\text {eq }}$ and $z_{\text {dur }}$ determines how smoothly this transition takes place. We define the following dimensionless energy density parameters for matter, radiation, neutrino and tachyon field, respectively,

$$
\begin{aligned}
\Omega_{m} & =\frac{\rho_{m}}{3 H^{2} M_{\mathrm{Pl}}^{2}} \\
\Omega_{r} & =\frac{\rho_{r}}{3 H^{2} M_{\mathrm{Pl}}^{2}} \\
\Omega_{\nu} & =\frac{\rho_{\nu}}{3 H^{2} M_{\mathrm{Pl}}^{2}} \\
\Omega_{\phi} & =\frac{\rho_{\phi}}{3 H^{2} M_{\mathrm{Pl}}^{2}}
\end{aligned}
$$

Figure 1 shows the evolution of density parameters for matter, radiation, neutrino and tachyon field. We have started the analysis of the evolution of Universe from the radiation dominated era followed by the matter dominated epoch. The Universe has recently entered the dark energy dominated epoch which, in this figure, is characterized by the non-minimal coupling of tachyon field with neutrino matter. In figure 2, we have shown the evolution of various equation-of-state parameters as the universe evolves. During the radiation dominated era, since the neutrinos are relativistic, the equation of state parameter for radiation as well as the neutrino matter is $1 / 3$. Neutrinos remain relativistic even after the radiation cease to dominate and have become non-relativistic only in the recent past. The equation-of-state parameters, $\omega_{\text {eff }}, \omega_{\phi}$ and $\omega_{\mathrm{DE}}$, approaches -1 at the present time since the universe is going through an accelerated expansion.

We define the following dimensionless quantities,

$$
\begin{array}{r}
x=\frac{\dot{\phi}}{\sqrt{2}}, \quad y=\frac{\sqrt{V}}{\sqrt{3} H M_{\mathrm{Pl}}}, \quad \Omega_{m}=\frac{\rho_{m}}{3 H^{2} M_{\mathrm{Pl}}^{2}}, \quad \Omega_{r}=\frac{\rho_{r}}{3 H^{2} M_{\mathrm{Pl}}^{2}}, \\
\lambda=-M_{\mathrm{Pl}} \frac{V^{\prime}}{V^{3 / 2}}, \quad \omega_{\nu}=\frac{1}{6}\left[1+\tanh \left(\frac{\ln (1+z)-z_{\mathrm{eq}}}{z_{\mathrm{dur}}}\right)\right]
\end{array}
$$




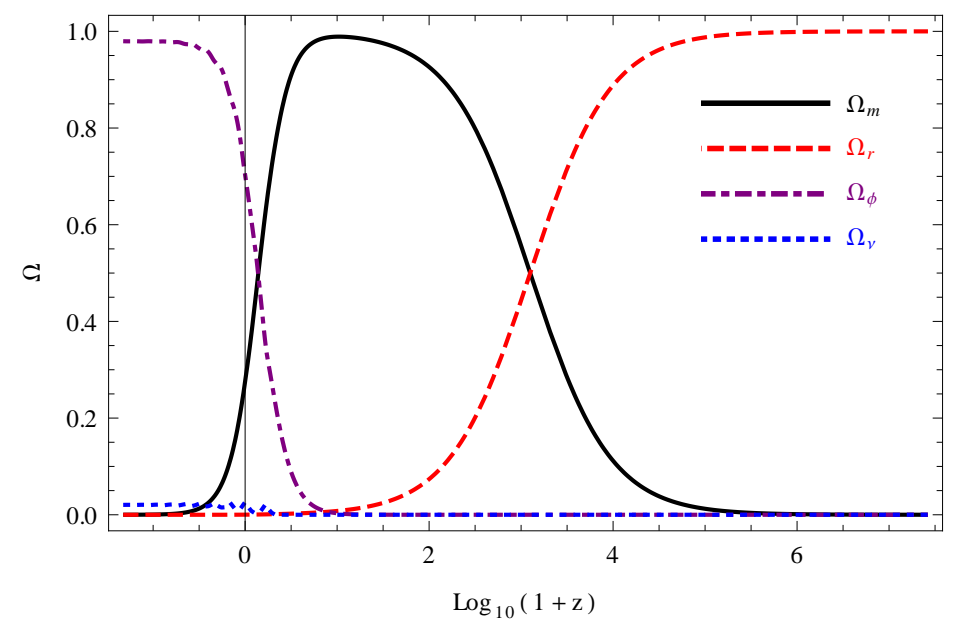

Figure 1. Figure shows the evolution of energy density parameters for radiation(red dashed line), matter(black solid line), tachyon field(purple dotted line) field and neutrino matter(blue dotdashed line), respectively, with respect to $\log _{10}(1+\mathrm{z})$. Here, we have considered $z_{\text {dur }}=3.0$.

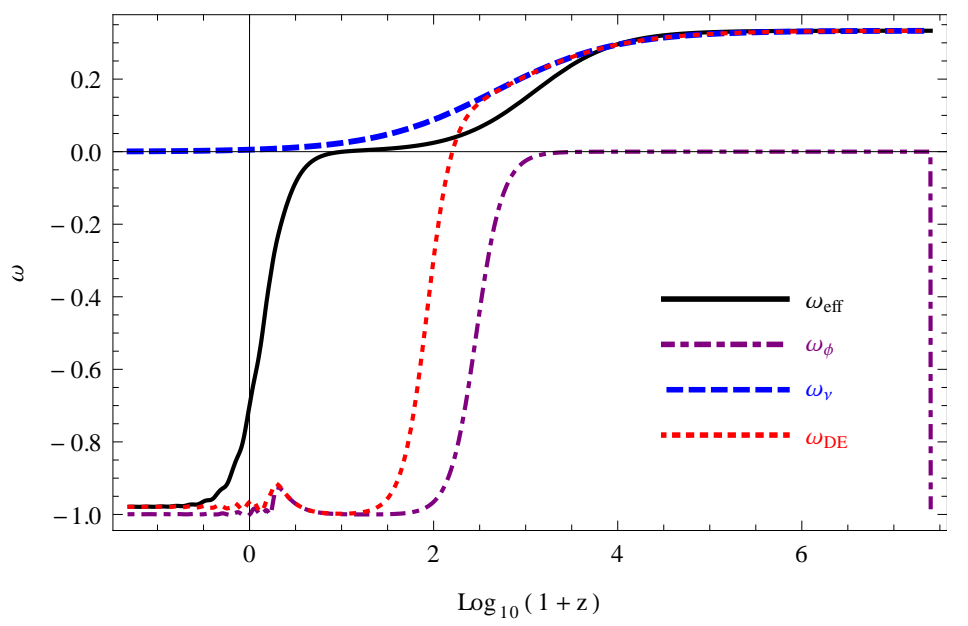

Figure 2. Figure shows the evolution of equation-of-state parameters, $\omega_{\text {eff }}$ (black solid line), $\omega_{\phi}$ (purple dotdashed line), $\omega_{\nu}$ (blue dashed line) and $\omega_{\mathrm{DE}}$ (red dotted line), respectively, with respect to $\log _{10}(1+\mathrm{z})$. We took $z_{\text {dur }}=3.0$ to plot this figure.

so that the cosmological equations in the autonomous form are written as,

$$
\begin{aligned}
\frac{\mathrm{d} x}{\mathrm{~d} N} & =\left(1-2 x^{2}\right)\left(-3 x+\sqrt{\frac{3}{2}} \lambda y+\sqrt{\frac{3}{2}} \frac{\sqrt{1-2 x^{2}}}{y} Q\left(3 \omega_{\nu}-1\right) \Omega_{\nu}\right), \\
\frac{\mathrm{d} y}{\mathrm{~d} N} & =\frac{y}{2}\left(3-\sqrt{6} \lambda x y-3 y^{2} \sqrt{1-2 x^{2}}+3 \omega_{\nu} \Omega_{\nu}+\Omega_{r}\right), \\
\frac{\mathrm{d} \Omega_{m}}{\mathrm{~d} N} & =\Omega_{m}\left(-3 y^{2} \sqrt{1-2 x^{2}}+3 \omega_{\nu} \Omega_{\nu}+\Omega_{r}\right), \\
\frac{\mathrm{d} \Omega_{r}}{\mathrm{~d} N} & =\Omega_{m}\left(-1-3 y^{2} \sqrt{1-2 x^{2}}+3 \omega_{\nu} \Omega_{\nu}+\Omega_{r}\right), \\
\frac{\mathrm{d} \omega_{\nu}}{\mathrm{d} N} & =\frac{2 \omega_{\nu}}{z_{\text {dur }}}\left(3 \omega_{\nu}-1\right)
\end{aligned}
$$


where, $N \equiv \ln a$, is the number of e-foldings.

The equation of state parameters in terms of the dimensionless variables can now be written as

$$
\begin{aligned}
\omega_{\phi} & =2 x^{2}-1, \\
\omega_{\text {eff }} & =-y^{2} \sqrt{1-2 x^{2}}+\omega_{\nu} \Omega_{\nu}+\frac{\Omega_{r}}{3}
\end{aligned}
$$

Energy density parameter for the tachyon field in terms of the dimensionless variables can be written as

$$
\Omega_{\phi}=\frac{y^{2}}{\sqrt{1-2 x^{2}}}
$$

and since

$$
\Omega_{m}+\Omega_{r}+\Omega_{\phi}+\Omega_{\nu}=1
$$

we can write the energy density parameter for neutrino matter as

$$
\Omega_{\nu}=1-\Omega_{m}+\Omega_{r}+\Omega_{\phi}
$$

Dark energy contribution comes from the tachyon field as well as the neutrino matter

$$
\Omega_{D E}=\Omega_{\phi}+\Omega_{\nu}
$$

Fixed points can be obtained by equating eq. (2.17)-(2.21) to zero. The fixed points for the system in which a tachyon field is non-minimally coupled to matter are derived in [87] and will be the same if we consider the case of tachyon field non-minimally coupled with neutrino matter. We are interested in the one which corresponds to the scaling solution, which as given in [87] is,

$$
x=\bar{x}, \quad y=\frac{3}{\sqrt{6}(\lambda+Q) \bar{x}}
$$

where, $\bar{x}$ satisfies the following relation

$$
\frac{\sqrt{1-2 \bar{x}^{2}}}{\bar{x}^{2}}=\frac{2 Q(\lambda+Q)}{3}
$$

The equation of state parameter for the tachyon field corresponding to the above fixed point is,

$$
\omega_{\phi}=2 \bar{x}^{2}-1=\frac{9}{2 Q^{2}(Q+\lambda)^{2}}\left[-1+\sqrt{1+\frac{4}{9} Q^{2}(Q+\lambda)^{2}}\right]-1
$$

Clearly, as $Q \rightarrow 0, x \rightarrow 1 / \sqrt{2}, y \rightarrow \sqrt{3} /(\lambda+Q), \omega_{\phi} \rightarrow 0$ while in the limit $Q \rightarrow \infty, x \rightarrow 0$, $y \rightarrow 1, \omega_{\phi} \rightarrow-1$. So, the accelerated expansion is realised for large $\mathrm{Q}$.

From eq. (2.6), it can be clearly seen that late time acceleration can only happen if $\dot{\phi}$ is very small. So in the limit when $\dot{\phi} \ll 1$, tachyon lagrangian (2.3) can be approximated at late times as,

$$
p_{\phi} \approx-V(\phi)+V(\phi) \frac{\dot{\phi}^{2}}{2}
$$

which can be written in the canonical form by redefining the field as

$$
V(\phi)=\left(\frac{\partial \sigma}{\partial \phi}\right)^{2},
$$


provided that the potential is very shallow at late times. Relation between $\phi$ and $\sigma$ can be obtained by integrating $(2.30)$

$$
\frac{\lambda \sigma}{2 M_{\mathrm{Pl}}}=\ln \phi-\ln C
$$

so that $V(\sigma)$ reads

$$
V(\sigma)=\frac{4 M_{\mathrm{Pl}}^{2}}{\lambda^{2} C^{2}} e^{-\lambda \sigma / M_{\mathrm{Pl}}}
$$

where $\mathrm{C}$ is the integration constant.

So, if at present time $\sigma=\sigma_{0}$ and $V(\sigma)=V\left(\sigma_{0}\right)$, then

$$
C^{2}=\frac{4 M_{\mathrm{Pl}}^{2}}{\lambda^{2} V\left(\sigma_{0}\right)} e^{-\lambda \sigma_{0} / M_{\mathrm{Pl}}}
$$

so that

$$
V(\sigma)=V\left(\sigma_{0}\right) e^{-\lambda\left(\sigma-\sigma_{0}\right) / M_{\mathrm{Pl}}}
$$

Eq. (2.29) becomes

$$
p_{\sigma}=\frac{\dot{\sigma}^{2}}{2}-V(\sigma),
$$

with equation of state parameter taking the form

$$
\omega_{\sigma}=\frac{\dot{\sigma}^{2}}{V}-1
$$

and equation of motion in terms of the new field $\sigma$ is

$$
\ddot{\sigma}+3 H \dot{\sigma}=-\frac{\partial V}{\partial \sigma}-\frac{Q}{M_{\mathrm{Pl}}} \rho_{\nu}
$$

considering the fact that neutrinos become non-relativistic at late times.

Now, if we define, $\tilde{\rho_{\nu}}=\rho_{\nu} e^{-Q\left(\sigma-\sigma_{0}\right) / M_{\mathrm{Pl}}}$, then the effective potential can be written as

$$
V_{\mathrm{eff}}(\sigma)=V(\sigma)+\tilde{\rho_{\nu}} e^{Q\left(\sigma-\sigma_{0}\right) / M_{\mathrm{P} 1}}
$$

which has a minimum at

$$
\sigma_{\min }=\sigma_{0}+\frac{M_{\mathrm{Pl}}}{Q+\lambda} \ln \left[\frac{V\left(\sigma_{0}\right) \lambda}{Q \tilde{\rho_{\nu}}}\right]
$$

which gives us the effective potential, $V_{\text {eff }}$ at the minimum

$$
V_{\text {eff }}\left(\sigma_{\min }\right)=V\left(\sigma_{\min }\right)\left[1+\frac{\lambda}{Q}\right]
$$

where,

$$
V\left(\sigma_{\min }\right)=\frac{Q}{\lambda} \rho_{\nu}\left(\sigma_{\min }\right)
$$

is the potential at minimum. Now, effective potential can be rewritten as

$$
V_{\mathrm{eff}}\left(\sigma_{\min }\right)=\Omega_{\nu}\left(\sigma_{\min }\right)\left[1+\frac{Q}{\lambda}\right] 3 H_{0}^{2} M_{\mathrm{Pl}}^{2}
$$

As given by the Planck 2015 results [114], the bound on neutrino matter is $\Omega_{\nu} \lesssim 0.005$ and the fact that field must settle down at the minimum of the effective potential in order to 


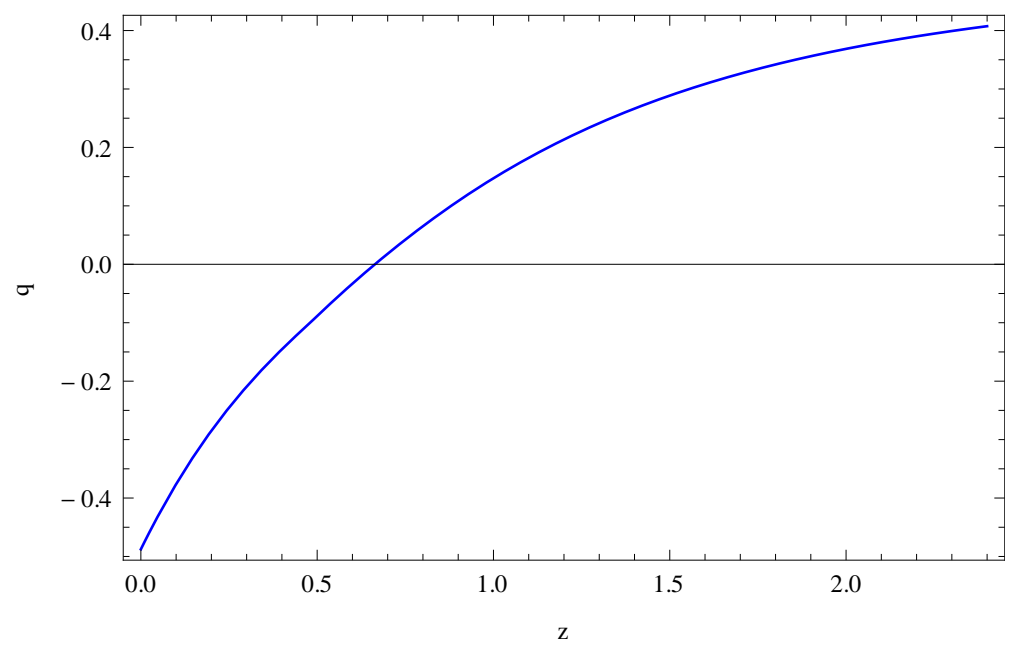

Figure 3. Figure shows the evolution of deceleration parameter, $q$ with respect to redshift $z$.

get the late time cosmic acceleration, $Q \gg \lambda$ for the effective potential to be of the order of $3 H_{0}^{2} M_{\mathrm{Pl}}^{2}$ at the minimum.

Furthermore, we have determined the cosmological deceleration-acceleration transition redshift [115-119]. The deceleration parameter, for the model under consideration, is defined as

$$
q \equiv-\frac{\ddot{a}}{a H^{2}}=\frac{1}{2}\left[\Omega_{m}+\Omega_{\phi}\left(1+3 \omega_{\phi}\right)+\Omega_{\nu}\left(1+3 \omega_{\nu}\right)+2 \Omega_{r}\right]
$$

The redshift at which the deceleration parameter, $q$, changes sign from positive to negative corresponds to the onset of late-time acceleration. Figure 3 shows the evolution of the deceleration parameter $q$ with respect to the redshift $z$. The redshift around which the transition from the decelerating expansion to the accelerating expansion occurs is found to be $z_{\mathrm{da}}=0.663$.

\subsection{Observational Constraints}

In figure 4, we have constrained our model parameters $(Q, \lambda)$ using Supernovae and BAO data. We have used the compilation of 580 data points of Union2.1 dataset [120] for SN1a observation and for BAO observation, we have used the data from [121]. The constraints on the model parameter $Q$ and $\lambda$ are obtained by marginalising $\chi^{2}$ over the value of $\Omega_{\nu 0}$. As can be seen from the figure, the values of $\lambda>0.72$ are ruled out at $1 \sigma$ level while from $2 \sigma$ confidence contour, the upper bound on $\lambda$ is 1.4 for large vales of $Q$ so that the ratio $Q / \lambda \gg 1$ which is required for the minimum of the effective potential to be of the order of $3 H_{0}^{2} M_{\mathrm{Pl}}^{2}$ at the present epoch. The dashed line in the plot corresponds to the redshift where $q=0$.

In figure 5, we have shown the evolution of normalized Hubble parameter for our model and compared it with that of the $\Lambda \mathrm{CDM}$ model. We have also plotted data points for normalized Hubble parameter, $H / H_{0}$ with $1 \sigma$ error bars which have been calculated from the compilation of 29 points of $\mathrm{H}(\mathrm{z})$ data $[115,116]$ using the present value of Hubble parameter, $H_{0}=(67.8 \pm 0.9) \mathrm{km} \mathrm{s}^{-1} \mathrm{Mpc}^{-1}$ from Planck 2015 results [114]. Error in $H / H_{0}$ can be 


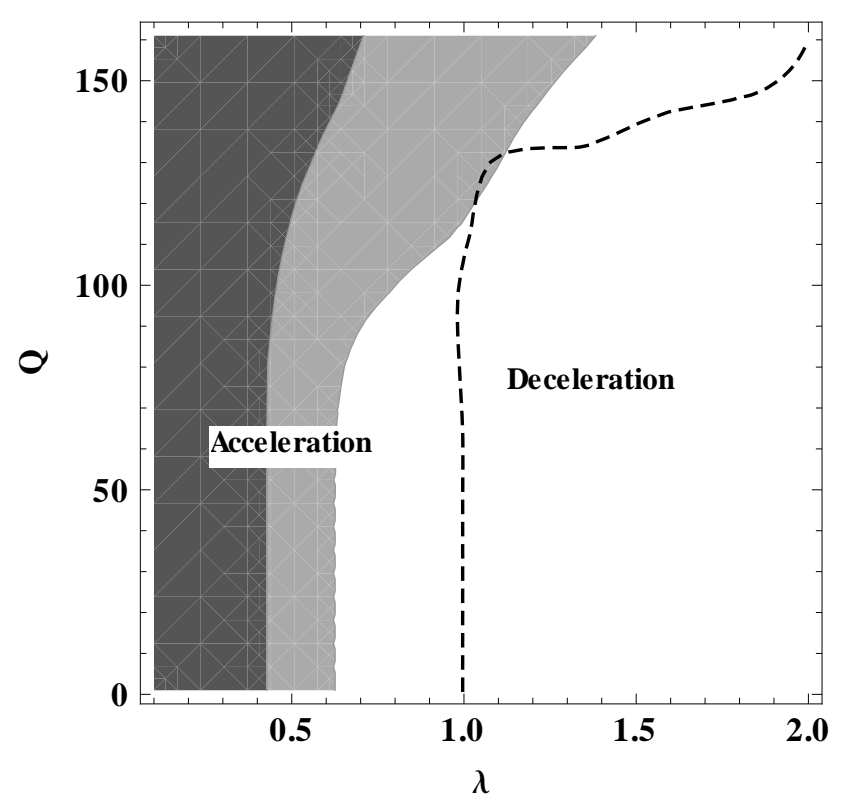

Figure 4. Figure shows the $1 \sigma$ (grey) and $2 \sigma$ (light grey) contours for the model parameters $Q$ and $\lambda$ using SN1a and BAO data. The dashed line corresponds to the onset of late-time cosmic acceleration. The $q=0$ dashed line clearly separates the decelerating and accelerating regimes.

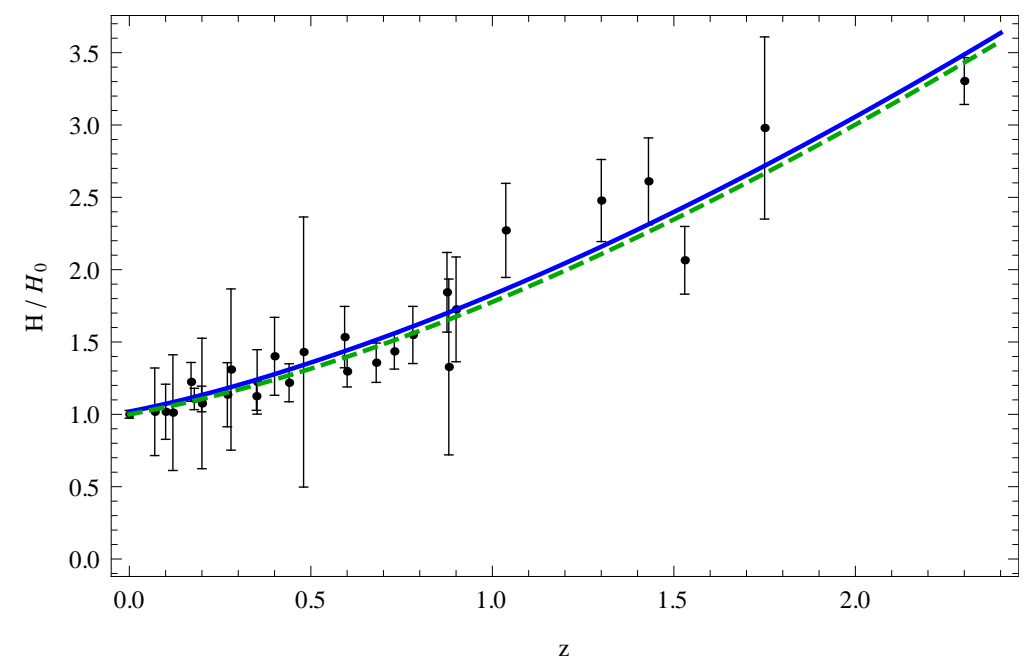

Figure 5. Figure shows the normalized Hubble parameter for the model being studied in this paper(blue solid line) and $\Lambda \mathrm{CDM}$ (green dashed line) with respect to the redshift, $z$. We have also plotted the normalized Hubble data with $1 \sigma$ error bars calculated from the compilation of $\mathrm{H}(\mathrm{z})$ data points $[122-128]$.

calculated [129] as

$$
\sigma_{\left(H / H_{0}\right)}=\left(\frac{\sigma_{H}}{H}+\frac{\sigma_{H_{0}}}{H_{0}}\right) \frac{H}{H_{0}}
$$

We thereby concludes that our model satisfies the observational constraints in the comfortable region of the parameter space $(Q, \lambda)$. 


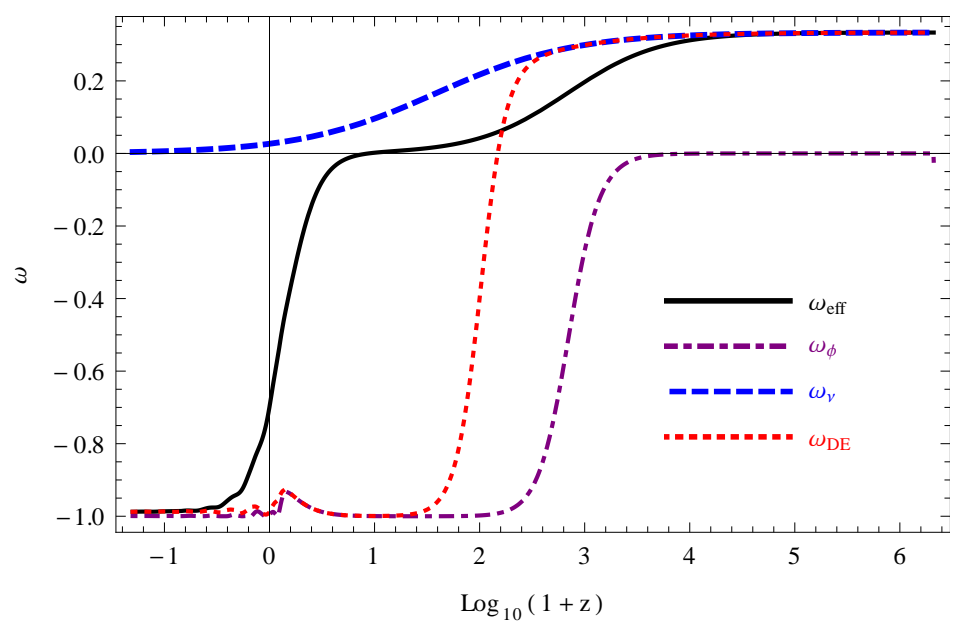

Figure 6. Figure shows the evolution of equation-of-state parameter with respect to $\log _{10}(1+\mathrm{z})$, for the case of string inspired exponential potential.

\section{Cosmology with string inspired Potential}

As mentioned in the introduction, the string inspired potential for rolling tachyon typically behaves as decaying exponential away from its maximum. In this case, the dark matter like solution with $\omega_{\phi}=0$ is a late-time attractor of the system [47]. In what follows, we now consider the case of exponential potential of tachyon field of the form

$$
V(\phi)=V_{0} \mathrm{e}^{-\alpha \phi}
$$

where $\alpha$ is a constant with mass dimension 1 and $\lambda$ is the dimensionless parameter which is associated with the slope of potential. In case of the exponential potential, we have

$$
\lambda \equiv-M_{\mathrm{Pl}} \frac{V^{\prime}}{V^{3 / 2}}=\frac{\alpha M_{\mathrm{Pl}}}{\sqrt{V}}
$$

which is not constant and hence does not give the scaling solution. Rolling tachyon with string inspired potential as such can not give rise to late-time acceleration. One might bring in a class of phenomenological potentials to do the job. In our opinion, it could still be of interest if the dynamics has generic features such as scaling behavior we considered before. As for the string inspired case, we could hope to get the desired late-time behavior by bringing in again the massive neutrino matter. Considering then the interaction between tachyon field and massive neutrino matter, we study the late-time dynamics for exponential potential by following the same procedure as above. So, in addition to the cosmological equations (2.17)-(2.21), we also have the evolution equation of $\lambda$,

$$
\frac{\mathrm{d} \lambda}{\mathrm{d} N}=\sqrt{\frac{3}{2}} \lambda^{2} x y
$$

Solving these equations numerically, the figure 6 shows that even though we get non-scaling solutions, the effective equation of state parameter approaches -1 at late times because of the coupling between tachyon field and neutrino matter. 


\section{Conclusion}

In this paper, we have investigated a system in which the tachyon field is non-minimally coupled to massive neutrino matter. In such a system, the equation of state parameter of neutrino matter is chosen phenomenologically such that neutrinos are relativistic for most of the history of universe and become non-relativistic only in the recent past. We have considered potentials that correspond to scaling as well as the non-scaling solutions. First, we have investigated the inverse square potential, $\phi^{-2}$, for which the slope, $\lambda=-M_{\mathrm{Pl}} V^{\prime} / V^{3 / 2}$ is a constant thereby the tachyon field exhibits a scaling behavior. However, in this case, the field can mimic a hypothetical background fluid with negative equation of state. We have shown that situation can be remedied by invoking non-minimal coupling to massive neutrino matter.

In presence of coupling, the scaling solution is an attractor of the system which mimics the desired equation of state for a very large value of coupling constant, $Q$. We numerically checked that $\dot{\phi} \ll 1$ in the asymptotic limit. In this limit, it is shown that the tachyon field is reduced to the canonical field at late times through a suitable transformation. Since tachyon is non-minimally coupled to neutrino matter and coupling builds up dynamically only at late stages, the effective potential of the field acquires a minimum at late times.

According to Planck 2015 results [114], the bound on present value of massive neutrino matter is $\Omega_{\nu} \lesssim 0.005$ which implies that coupling should be large for the field to settle down at the minimum of the potential around the present epoch(as shown in eq. (2.42)). We have analytically demonstrated that late time cosmic acceleration can be realized if the tachyon field is coupled non-minimally to the massive neutrino matter thereby leaving the matter dominated epoch intact. We have also shown the $1 \sigma$ and $2 \sigma$ confidence level in the parameter space $(Q, \lambda)$ using $\mathrm{SN1} 1 \mathrm{a}$ and $\mathrm{BAO}$ data. The normalized Hubble parameter for $\Lambda \mathrm{CDM}$ and our model have also been plotted and we have compared that with the $\mathrm{H}(\mathrm{z})$ data. Secondly, we have also examined the case of string inspired exponential potential that does not give rise to the scaling solution for the model under consideration. Indeed, in this case, the dark matter like solution is a late-time attractor of the system. We have demonstrated that due to the coupling between tachyon and massive neutrino matter, $\omega_{\text {eff }}$ approaches -1 at late times as shown in figure 6 . We therefore conclude that rolling tachyon with and without scaling potential can successfully give rise to late time acceleration by invoking non-minimal coupling with massive neutrino matter.

\section{ACKNOWLEDGMENTS}

We are thankful to M. Sami and Md. Wali Hossain for useful discussions. Safia Ahmad acknowledges DST, Govt. of India for financial support through Inspire Fellowship (DST/INSPIRE Fellowship/2012/614).

\section{References}

[1] A. G. Riess et al. [Supernova Search Team Collaboration], Astron. J. 116, 1009 (1998) [astro-ph/9805201].

[2] S. Perlmutter et al. [Supernova Cosmology Project Collaboration], Astrophys. J. 517, 565 (1999) [astro-ph/9812133].

[3] J. L. Tonry et al. [Supernova Search Team Collaboration], Astrophys. J. 594, 1 (2003) [astro-ph/0305008]. 
[4] B. Ratra and P. J. E. Peebles, Phys. Rev. D 37, 3406 (1988). doi:10.1103/PhysRevD.37.3406

[5] P. J. E. Peebles and B. Ratra, Astrophys. J. 325, L17 (1988). doi:10.1086/185100

[6] E. J. Copeland, M. Sami and S. Tsujikawa, Int. J. Mod. Phys. D 15, 1753 (2006) [hep-th/0603057].

[7] V. Sahni and A. A. Starobinsky, Int. J. Mod. Phys. D 9, 373 (2000) [astro-ph/9904398].

[8] J. Frieman, M. Turner and D. Huterer, Ann. Rev. Astron. Astrophys. 46, 385 (2008) [arXiv:0803.0982 [astro-ph]].

[9] T. Padmanabhan, Phys. Rept. 380, 235 (2003) [hep-th/0212290].

[10] T. Padmanabhan, AIP Conf. Proc. 861, 179 (2006) [astro-ph/0603114].

[11] V. Sahni and A. Starobinsky, Int. J. Mod. Phys. D 15, 2105 (2006) [astro-ph/0610026].

[12] P. J. E. Peebles and B. Ratra, Rev. Mod. Phys. 75, 559 (2003) [astro-ph/0207347].

[13] L. Perivolaropoulos, AIP Conf. Proc. 848, 698 (2006) [astro-ph/0601014].

[14] M. Sami, arXiv:0901.0756 [hep-th].

[15] M. Sami, Curr. Sci. 97, 887 (2009) [arXiv:0904.3445 [hep-th]].

[16] M. Sami and R. Myrzakulov, arXiv:1309.4188 [hep-th].

[17] R. R. Caldwell, Phys. Lett. B 545, 23 (2002) [astro-ph/9908168].

[18] R. R. Caldwell, M. Kamionkowski and N. N. Weinberg, Phys. Rev. Lett. 91, 071301 (2003) [astro-ph/0302506].

[19] S. M. Carroll, M. Hoffman and M. Trodden, Phys. Rev. D 68, 023509 (2003) [astro-ph/0301273].

[20] P. Singh, M. Sami and N. Dadhich, Phys. Rev. D 68, 023522 (2003) [hep-th/0305110].

[21] J. g. Hao and X. z. Li, Phys. Rev. D 68, 043501 (2003) [arXiv:hep-th/0305207].

[22] A. Sen, JHEP 0204, 048 (2002) [hep-th/0203211].

[23] A. Sen, JHEP 0207, 065 (2002) [hep-th/0203265].

[24] A. Sen, Phys. Scripta T 117, 70 (2005) [hep-th/0312153].

[25] A. Mazumdar, S. Panda and A. Perez-Lorenzana, Nucl. Phys. B 614, 101 (2001) [hep-ph/0107058].

[26] E. J. Copeland, M. R. Garousi, M. Sami and S. Tsujikawa, Phys. Rev. D 71, 043003 (2005) [hep-th/0411192].

[27] J. M. Aguirregabiria and R. Lazkoz, Phys. Rev. D 69, 123502 (2004) [hep-th/0402190].

[28] G. W. Gibbons, Phys. Lett. B 537, 1 (2002) [hep-th/0204008].

[29] T. Padmanabhan, Phys. Rev. D 66, 021301 (2002) [hep-th/0204150].

[30] J. S. Bagla, H. K. Jassal and T. Padmanabhan, Phys. Rev. D 67, 063504 (2003) [astro-ph/0212198].

[31] T. Padmanabhan and T. R. Choudhury, Phys. Rev. D 66, 081301 (2002) [hep-th/0205055].

[32] A. V. Frolov, L. Kofman and A. A. Starobinsky, Phys. Lett. B 545, 8 (2002) [hep-th/0204187].

[33] R. Rangdee and B. Gumjudpai, Astrophys. Space Sci. 349, 975 (2014) [arXiv:1210.5550 [astro-ph.CO]].

[34] J. G. Hao and X. z. Li, Phys. Rev. D 66, 087301 (2002) [hep-th/0209041].

[35] V. Gorini, A. Y. Kamenshchik, U. Moschella and V. Pasquier, Phys. Rev. D 69, 123512 (2004) [hep-th/0311111]. 
[36] L. P. Chimento, Phys. Rev. D 69, 123517 (2004) [astro-ph/0311613].

[37] M. B. Causse, astro-ph/0312206.

[38] L. R. Abramo, F. Finelli and T. S. Pereira, Phys. Rev. D 70, 063517 (2004) [astro-ph/0405041].

[39] S. K. Srivastava, gr-qc/0409074.

[40] R. Yang and J. Qi, Eur. Phys. J. C 72, 2095 (2012) [arXiv:1205.5968 [gr-qc]].

[41] V. K. Shchigolev and M. P. Rotova, Mod. Phys. Lett. A 27, 1250086 (2012) [arXiv:1203.5030 [gr-qc]].

[42] E. Elizalde and J. Q. Hurtado, Int. J. Mod. Phys. D 14, 1439 (2005) [gr-qc/0412106].

[43] R. C. G. Landim, Int. J. Mod. Phys. D 24, no. 11, 1550085 (2015) [arXiv:1505.03243 [hep-th]].

[44] R. C. G. Landim, arXiv:1507.00902 [gr-qc].

[45] M. Fairbairn and M. H. G. Tytgat, Phys. Lett. B 546, 1 (2002) [hep-th/0204070].

[46] D. Choudhury, D. Ghoshal, D. P. Jatkar and S. Panda, Phys. Lett. B 544, 231 (2002) [hep-th/0204204].

[47] M. Sami, P. Chingangbam and T. Qureshi, Phys. Rev. D 66, 043530 (2002) [hep-th/0205179].

[48] M. Sami, Mod. Phys. Lett. A 18, 691 (2003) [hep-th/0205146].

[49] A. Feinstein, Phys. Rev. D 66, 063511 (2002) [hep-th/0204140].

[50] G. Shiu and I. Wasserman, Phys. Lett. B 541, 6 (2002) [hep-th/0205003].

[51] L. Kofman and A. D. Linde, JHEP 0207, 004 (2002) [hep-th/0205121].

[52] G. Shiu, S. H. H. Tye and I. Wasserman, Phys. Rev. D 67, 083517 (2003) [hep-th/0207119].

[53] J. c. Hwang and H. Noh, Phys. Rev. D 66, 084009 (2002) [hep-th/0206100].

[54] Y. S. Piao, R. G. Cai, X. m. Zhang and Y. Z. Zhang, Phys. Rev. D 66, 121301 (2002) [hep-ph/0207143].

[55] Y. S. Piao, Q. G. Huang, X. m. Zhang and Y. Z. Zhang, Phys. Lett. B 570, 1 (2003) [hep-ph/0212219].

[56] J. M. Cline, H. Firouzjahi and P. Martineau, JHEP 0211, 041 (2002) [hep-th/0207156].

[57] S. Mukohyama, Phys. Rev. D 66, 123512 (2002) [hep-th/0208094].

[58] G. N. Felder, L. Kofman and A. Starobinsky, JHEP 0209, 026 (2002) [hep-th/0208019].

[59] M. C. Bento, O. Bertolami and A. A. Sen, Phys. Rev. D 67, 063511 (2003) [hep-th/0208124].

[60] X. z. Li, D. j. Liu and J. g. Hao, J. Shanghai Normal Univ. (Natural Sciences), Vol. 33(4), (2004), 29 [hep-th/0207146].

[61] C. j. Kim, H. B. Kim and Y. b. Kim, Phys. Lett. B 552, 111 (2003) [hep-th/0210101].

[62] T. Matsuda, Phys. Rev. D 67, 083519 (2003) [hep-ph/0302035].

[63] A. Das and A. DeBenedictis, Gen. Rel. Grav. 36, 1741 (2004) [gr-qc/0304017].

[64] Z. K. Guo, Y. S. Piao, R. G. Cai and Y. Z. Zhang, Phys. Rev. D 68, 043508 (2003) [hep-ph/0304236].

[65] G. W. Gibbons, Class. Quant. Grav. 20, S321 (2003) [hep-th/0301117].

[66] M. Majumdar and A. C. Davis, Phys. Rev. D 69, 103504 (2004) [hep-th/0304226].

[67] S. Nojiri and S. D. Odintsov, Phys. Lett. B 571, 1 (2003) [hep-th/0306212].

[68] E. Elizalde, J. E. Lidsey, S. Nojiri and S. D. Odintsov, Phys. Lett. B 574, 1 (2003) [hep-th/0307177]. 
[69] M. R. Garousi, M. Sami and S. Tsujikawa, Phys. Rev. D 70, 043536 (2004) [hep-th/0402075].

[70] M. Sami, P. Chingangbam and T. Qureshi, Pramana 62, 765 (2004) [hep-th/0301140].

[71] M. R. Garousi, M. Sami and S. Tsujikawa, Phys. Lett. B 606, 1 (2005) [hep-th/0405012].

[72] G. N. Felder and L. Kofman, Phys. Rev. D 70, 046004 (2004) [hep-th/0403073].

[73] G. Calcagni, Phys. Rev. D 70, 103525 (2004) [hep-th/0406006].

[74] J. Raeymaekers, JHEP 0410, 057 (2004) [hep-th/0406195].

[75] G. Calcagni and S. Tsujikawa, Phys. Rev. D 70, 103514 (2004) [astro-ph/0407543].

[76] N. Barnaby and J. M. Cline, Int. J. Mod. Phys. A 19, 5455 (2004) [hep-th/0410030].

[77] S. Panda, M. Sami and S. Tsujikawa, Phys. Rev. D 73, 023515 (2006) [hep-th/0510112].

[78] K. L. Panigrahi, Phys. Lett. B 601, 64 (2004) [hep-th/0407134].

[79] A. Ghodsi and A. E. Mosaffa, Nucl. Phys. B 714, 30 (2005) [hep-th/0408015].

[80] G. Shiu, hep-th/0210313.

[81] B. C. Paul and M. Sami, Phys. Rev. D 70, 027301 (2004) [hep-th/0312081].

[82] D. A. Steer and F. Vernizzi, Phys. Rev. D 70, 043527 (2004) [hep-th/0310139].

[83] K. Nozari and N. Rashidi, Phys. Rev. D 88, no. 2, 023519 (2013) [arXiv:1306.5853 [gr-qc]].

[84] S. Mukohyama, Phys. Rev. D 66, 024009 (2002) [hep-th/0204084].

[85] N. Barbosa-Cendejas, J. De-Santiago, G. German, J. C. Hidalgo and R. R. Mora-Luna, arXiv:1506.09172 [astro-ph.CO].

[86] S. Tsujikawa and M. Sami, Phys. Lett. B 603, 113 (2004) [hep-th/0409212]

[87] B. Gumjudpai, T. Naskar, M. Sami and S. Tsujikawa, JCAP 0506, 007 (2005)

[88] C. Wetterich, Phys. Rev. D 89, 024005 (2014) [arXiv:1308.1019 [astro-ph.CO]].

[89] C. Wetterich, Physics of the Dark Universe (2013), [arXiv:1303.6878 [astro-ph.CO]].

[90] C. Wetterich, Phys. Lett. B 726, 15 (2013) [arXiv:1303.4700 [astro-ph.CO]].

[91] C. Wetterich, arXiv:1401.5313 [astro-ph.CO].

[92] C. Wetterich, arXiv:1402.5031 [astro-ph.CO].

[93] L. Amendola, M. Baldi and C. Wetterich, Phys. Rev. D 78, 023015 (2008) [arXiv:0706.3064 [astro-ph]].

[94] C. Wetterich, Phys. Lett. B 655, 201 (2007) [arXiv:0706.4427 [hep-ph]].

[95] V. Pettorino, N. Wintergerst, L. Amendola and C. Wetterich, Phys. Rev. D 82, 123001 (2010) [arXiv: 1009.2461[astro-ph.CO]].

[96] R. Fardon, A. E. Nelson and N. Weiner, JCAP 0410, 005 (2004) [astro-ph/0309800].

[97] X. -J. Bi, P. -h. Gu, X. -l. Wang and X. -m. Zhang, Phys. Rev. D 69, 113007 (2004) [hep-ph/0311022].

[98] P. Q. Hung and H. Pas, Mod. Phys. Lett. A 20, 1209 (2005) [astro-ph/0311131].

[99] R. D. Peccei, Phys. Rev. D 71, 023527 (2005) [hep-ph/0411137].

[100] X. -J. Bi, B. Feng, H. Li and X. -m. Zhang, Phys. Rev. D 72, 123523 (2005) [hep-ph/0412002].

[101] A. W. Brookfield, C. van de Bruck, D. F. Mota and D. Tocchini-Valentini, Phys. Rev. Lett. 96, 061301 (2006) [astro-ph/0503349].

[102] A. W. Brookfield, C. van de Bruck, D. F. Mota and D. Tocchini-Valentini, Phys. Rev. D 73, 083515 (2006) [Erratum-ibid. D 76, 049901 (2007)] [astro-ph/0512367]. 
[103] O. E. Bjaelde, A. W. Brookfield, C. van de Bruck, S. Hannestad, D. F. Mota, L. Schrempp and D. Tocchini-Valentini, JCAP 0801, 026 (2008) [arXiv:0705.2018 [astro-ph]].

[104] N. Afshordi, M. Zaldarriaga and K. Kohri, Phys. Rev. D 72, 065024 (2005) [astro-ph/0506663].

[105] D. F. Mota, V. Pettorino, G. Robbers and C. Wetterich, Phys. Lett. B 663, 160 (2008) [arXiv:0802.1515 [astro-ph]].

[106] G. La Vacca and D. F. Mota, Astron. Astrophys. 560, A53 (2013) [arXiv:1205.6059 [astro-ph.CO]].

[107] L. G. Collodel and G. M. Kremer, Grav. Cosmol. 18, 196 (2012) [arXiv:1203.3061 [gr-qc]].

[108] H. Motohashi, A. A. Starobinsky and J. Yokoyama, Phys. Rev. Lett. 110, no. 12, 121302 (2013) [arXiv:1203.6828 [astro-ph.CO]].

[109] A. S. Chudaykin, D. S. Gorbunov, A. A. Starobinsky and R. A. Burenin, JCAP 1505, no. 05, 004 (2015) [arXiv:1412.5239 [astro-ph.CO]].

[110] M. W. Hossain, R. Myrzakulov, M. Sami and E. N. Saridakis, Phys. Rev. D 90, 023512 (2014) [1402.6661 [gr-qc]].

[111] M. Wali Hossain, R. Myrzakulov, M. Sami and E. N. Saridakis, Int. J. Mod. Phys. D 24, no. 05, 1530014 (2015) [arXiv:1410.6100 [gr-qc]].

[112] F. Piazza and S. Tsujikawa, JCAP 0407, 004 (2004) [hep-th/0405054]

[113] L. Amendola, Phys. Rev. D 62, 043511 (2000) [astro-ph/9908023]. [hep-th/0502191]

[114] P. A. R. Ade et al. [Planck Collaboration], arXiv:1502.01589 [astro-ph.CO].

[115] O. Farooq, S. Crandall and B. Ratra, Phys. Lett. B 726, 72 (2013) doi:10.1016/j.physletb.2013.08.078 [arXiv:1305.1957 [astro-ph.CO]].

[116] O. Farooq and B. Ratra, Astrophys. J. 766, L7 (2013) doi:10.1088/2041-8205/766/1/L7 [arXiv:1301.5243 [astro-ph.CO]].

[117] O. Farooq, D. Mania and B. Ratra, Astrophys. J. 764, 138 (2013) doi:10.1088/0004-637X/764/2/138 [arXiv:1211.4253 [astro-ph.CO]].

[118] O. Farooq, D. Mania and B. Ratra, Astrophys. Space Sci. 357, no. 1, 11 (2015) doi:10.1007/s10509-015-2319-2 [arXiv:1308.0834 [astro-ph.CO]].

[119] S. Capozziello, O. Farooq, O. Luongo and B. Ratra, Phys. Rev. D 90, no. 4, 044016 (2014) doi:10.1103/PhysRevD.90.044016 [arXiv:1403.1421 [gr-qc]].

[120] N. Suzuki et al., Astrophys. J. 746, 85 (2012) [arXiv:1105.3470 [astro-ph.CO]].

[121] R. Giostri, M. V. d. Santos, I. Waga, R. R. R. Reis, M. O. Calvao and B. L. Lago, JCAP 1203, 027 (2012) [arXiv:1203.3213 [astro-ph.CO]] and the references therein for BAO data.

[122] J. Simon, L. Verde and R. Jimenez, Phys. Rev. D 71, 123001 (2005) [astro-ph/0412269].

[123] D. Stern, R. Jimenez, L. Verde, M. Kamionkowski and S. A. Stanford, JCAP 1002, 008 (2010) [arXiv:0907.3149 [astro-ph.CO]].

[124] C. Zhang, H. Zhang, S. Yuan, T. -J. Zhang and Y. -C. Sun, arXiv:1207.4541 [astro-ph.CO].

[125] M. Moresco, L. Verde, L. Pozzetti, R. Jimenez and A. Cimatti, JCAP 1207, 053 (2012) [arXiv:1201.6658 [astro-ph.CO]].

[126] C. -H. Chuang and Y. Wang, arXiv:1209.0210 [astro-ph.CO].

[127] C. Blake, S. Brough, M. Colless, C. Contreras, W. Couch, S. Croom, D. Croton and T. Davis et al., Mon. Not. Roy. Astron. Soc. 425, 405 (2012) [arXiv:1204.3674 [astro-ph.CO]]. 
[128] N. G. Busca, T. Delubac, J. Rich, S. Bailey, A. Font-Ribera, D. Kirkby, J. M. Le Goff and M. M. Pieri et al., Astron. Astrophys. 552, A96 (2013) [arXiv:1211.2616 [astro-ph.CO]].

[129] K. Bamba, M. W. Hossain, R. Myrzakulov, S. Nojiri and M. Sami, Phys. Rev. D 89, no. 8, 083518 (2014) doi:10.1103/PhysRevD.89.083518 [arXiv:1309.6413 [hep-th]]. 